\title{
Simulated Patient for Orthognathic Surgery
}

\author{
Horace H. S. Ip, Christy S. B. Kot and James Xia \\ Image Computing Group, Department of Computer Science \\ City University of Hong Kong \\ 83, Tat Chee Avenue, Kowloon, Hong Kong \\ E-mail: cship@cityu.edu.hk
}

\begin{abstract}
Orthognathic surgery corrects a wide range of minor and major facial and jaw irregularities. This surgery will improve the patients' ability to chew, speak and breathe. In many cases, a better appearance will also result. With the recent advances in virtual reality (VR) and three-dimensional (3D) medical imaging technology, orthognathic surgery simulations typically requires costly volumetric data acquisition modalities such CT or MRI imaging for patient modeling. In this paper, we presents an approach for constructing $3 D$ hard and soft tissue models of a patient based on colour portraits and conventional radiographs. This allows patient modeling to be done efficiently on low-cost platforms. Specifically, we extend the techniques developed by the author in [2] to hard tissue modeling. The extended technique employs a user-assisted approach to obtain the $3 D$ coordinates of the feature points of the human face and jaw respectively from conventional photographs and radiographs. Then the displacement vectors of the feature points are computed by correspondence matching and interpolation against a generic head model and jaw bone model. The resulting combined hard and soft tissue models can be used for orthognathic surgical planning on a low-cost, PCbased platform.
\end{abstract}

\section{Introduction}

At present, there are a number of commercial products of interactive orthognathic surgical simulation systems such as Dentofacial Planner Plus [7], which is developed by Dentofacial Software Inc., and CephScan, which is developed by CephScan Professional Software Products Inc. [8]. These systems use the cephalometric radiographs as the data source. However, the post-operative effect of the patient can only be viewed in two-dimensional (2D). Because of this limitation, a prototype of an interactive 3D orthognathic surgical simulation system was developed by Swiss Federal Institute of Technology and the Department of Maxillofacial Surgery of University Hospital in 1996 [9]. In 1998, a computer assisted 3D virtual osteotomy system for orthognathic surgery (CAVOS) on a PC-based platform was developed at the University of Hong Kong [3]. Both systems use computed tomography (CT) as the data source and the post-operative can be viewed in arbitrary views.

An individualized head model is a crucial element for an orthognathic surgical simulation system, previous approaches typically constructs individualized head model for the soft and hard tissues based on CT and MRI volumetric data. These systems are costly due to the high costs of the imaging (data acquisition) modalities and the high processing requirements of the resulting large quantity of $3 \mathrm{D}$ volumetric data. In this paper, we adopt a new approach that constructs 3D hard and soft tissue models based on the patient's portraits and skull radiographs. This allows orthognathic surgical planning to be done effectively on low-cost platforms.

\section{Three-dimensional Photo-realistic Textured Facial Model Generation}

The method for constructing an individualized 3D head model requires a 3D generic head model and portraits of two orthogonal views. In order to have a better quality of texture, it is recommended that two portraits of the frontal and lateral views should be taken at the same time. The application of constant light source will minimize the shadow reflected on the face. 

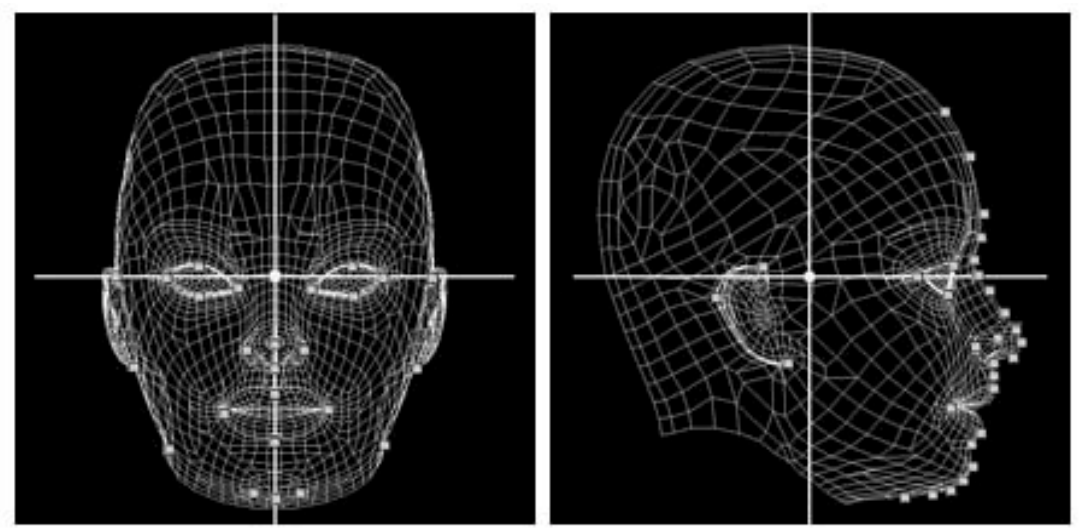

D - origin of the model

- feature vertices

Figure 1. Feature vertices definition for frontal and lateral view of the generic head model

\subsection{Generic Head Model and Facial Feature Vertices Definitions}

A generic polygonal head model, which contains 3118 polygons and 2953 vertices, is adopted in this implementation. There are certain basic feature points on the human face that characterize the eyes, nose, mouth, face shape, etc. For the two views of the generic head model, a set of feature vertices, that are used to modify the generic model directly, is defined. As shown in Figure 1, 25 feature vertices are defined for the front view of the generic head model and 30 feature vertices are defined for the side view.

\subsection{Image Normalization}

In order to align the facial features such as eyes, nose and mouth in the two views, all the input images are normalized as follows. [2]

$$
x=k\left(\frac{X}{H}\right) \quad y=k\left(\frac{Y}{H}\right)
$$

where

$x, y=$ normalized coordinates of the facial features

$k=$ scale factor

$X, Y=$ original coordinates of the facial features

$H=$ the facial length from the top of the head to the chin

\subsection{Facial Feature Vertices Identification}

Our semi-automatic scheme for constructing a 3D individualized head model employs a user-assisted technique to obtain the $3 \mathrm{D}$ coordinates of the facial feature points. The user is required to mark the origin and the feature vertices on the frontal and lateral views of the portraits respectively according to the feature vertices definitions of the generic head model. These feature vertices can be identified manually efficiently. It typically requires 50 seconds to mark all the feature vertices for both views. Figure 2 shows the feature vertices marked on the portraits of the two views.
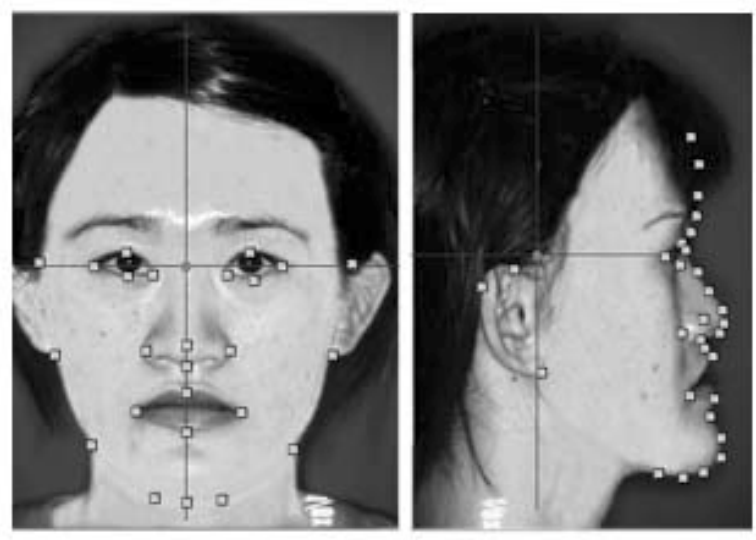

- - origin of the model

口- - feature vertices

Figure 2. Feature vertices marked on front view and side view of the portraits

\subsection{Computation of Feature Vertices Displacement Vectors}

After marking the feature vertices for an individual face, for each view of the face, a set of displacement vectors will be calculated by matching the feature vertices on the portraits to those vertices of the generic head model.

The displacement vector, denoted by DV in this paper, is defined as a vector from the position in 
generic model to the corresponding position in the portrait of the same view [2]. For each view, the DV of each feature vertex $\mathbf{n}$ will be defined as follows.

$$
D V_{n}^{f}=P_{n}^{f}-V_{n}^{f}
$$

where

$$
\begin{aligned}
& P=\text { a position vector of } \mathbf{n} \text { th feature point in the } \\
& \quad \text { generic head model } \\
& V=\text { a corresponding position vector of the } \mathbf{n} \text { th } \\
& \quad \text { feature point in the portrait } \\
& F=\text { number of feature vertices of generic model }
\end{aligned}
$$

From the DVs of the feature vertices, we can derive the DVs of the non-feature vertices by interpolation.

Having obtained the correspondence between the 2 sets of feature vertices identified on the frontal and lateral views of the patient respectively, we compute a set of displacement vectors, denoted by $\mathrm{DV}_{\mathrm{k}}$, that contains the DVs of feature vertices only. For a nonfeature vertex $P_{m}$ of the generic head model, we search for $\mathrm{N}$ nearest vertices within $\mathrm{DV}_{\mathrm{k}}$ along the directions of upward, downward, leftward and rightward. Then the distance between $\mathrm{P}_{\mathrm{m}}$ and every selected nearest vertex will be calculated and be denoted by $d_{m, n}$ (where $\mathrm{n}=1,2, \ldots \mathrm{N}$ ). Afterwards, the $\mathrm{N}$ nearest vertices will be rearranged in ascending order according to their distance to $\mathrm{P}_{\mathrm{m}}$. A weight function $\mathbf{w}$, that has a larger output of weight value for a smaller input of $d_{m, n}$, will be calculated as follows.

$$
w\left(d_{(m, n)}\right)=\frac{d_{m, n^{\prime}}}{{ }_{n=1}^{N} d_{m, n}}
$$

where

$$
n^{\prime}=(\mathrm{N}+1)-\mathrm{n} \quad(\mathrm{n}=1,2, \ldots \mathrm{N})
$$

This equation gives the weight function $\mathbf{w}$ for all the $\mathrm{N}$ nearest vertices. The $\mathrm{DV}_{\mathrm{m}}$ for a non-feature vertex $\mathrm{P}_{\mathrm{m}}$ can then be estimated by:

$$
D V^{m}={ }_{n=1}^{N}\left(w\left(d_{m, n}\right) \cdot D V_{k}^{n}\right)
$$

For every $\mathrm{DV}^{\mathrm{m}}$ of non-feature vertex that is found, it will be immediately added to the set $\mathrm{DV}_{\mathrm{k}}$ and it can be used subsequently for the estimation of the remaining non-feature vertices. This iterative search process continues until all the DVs of the non-feature vertices are found.
The above procedure is applied to the frontal and lateral views of the face independently. The resulting two sets of DVs are the set of displacement vectors for all the vertices on the $X-Y$ and $X-Z$ planes respectively. After calculating all the DVs, the new two-dimensional (2D) coordinates for every vertex on the frontal and lateral views are generated. The resulting $2 \mathrm{D}$ polygonal models of the two views of the patient are superimposed on to the portraits respectively as shown in Figure 3.
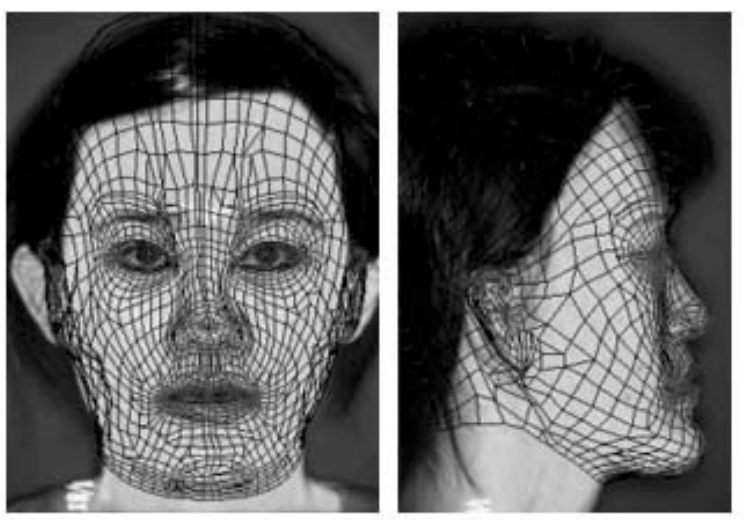

Figure 3. The alignment of the computer generated individualized head model and the original face images for the two views

\subsection{Individualized 3D Head Model Generation}

Given the two sets of 2D coordinates of the computer constructed head model for the frontal and lateral views, the individualized 3D head model can then be generated as follows:

$$
(x, y, z)=\left(x_{f}, \frac{y_{f}+y_{s}}{2}, z_{s}\right)
$$

where

$$
\begin{aligned}
& x, y, z=3 \mathrm{D} \text { coordinates of the } 3 \mathrm{D} \text { head model } \\
& x_{f}, y_{f}=2 \mathrm{D} \text { coordinates of the frontal view } \\
& x_{s}, y_{s}=2 \mathrm{D} \text { coordinates of the lateral view }
\end{aligned}
$$

As the generic model is adjusted with the frontal and lateral views separately, the values of $y_{f}$ and $y_{s}$ may not be the same, and the average of $y_{f}$ and $y_{s}$ is taken as final y. Figure 4 shows the computer constructed head model for the patient in arbitrary views. 


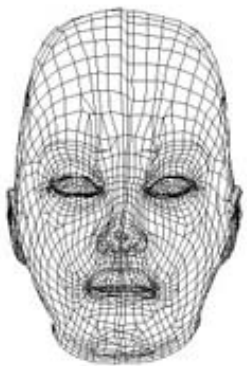

$0^{\circ}$

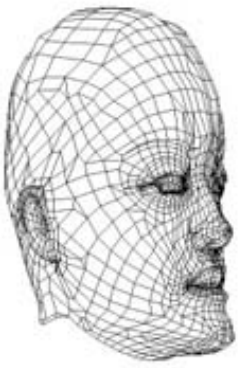

$60^{\circ}$

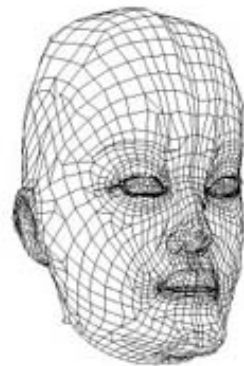

$30^{\circ}$

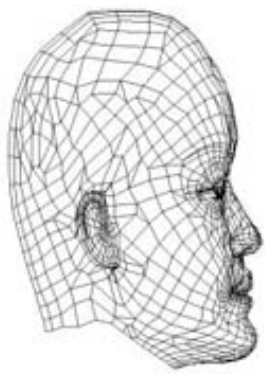

$90^{\circ}$
Figure 4. Different views of individualized 3D head model

\subsection{D Photo-realistic Textured Facial Model Generation}

Using OpenGL [6], the facial image texture from the two portraits can be mapped to $3 \mathrm{D}$ head model by indicating the $2 \mathrm{D}$ coordinates of the polygon on the portraits which are related to their corresponding 3D coordinates on the head model. Every polygon on the portrait of the front view is represented by the $\mathrm{x}$ and $\mathrm{y}$ coordinates of the head model while the $\mathrm{z}$ and $\mathrm{y}$ coordinates represent the location of polygon on the portrait of the side view. Figure 5 shows different views of the texture mapped 3D head model.

\section{Three-dimensional Individualized Jaw Model Generation}

Applying similar techniques to hard tissues, ie. bone structure, we are able to construct a 3D jaw bone model of a patient from his/her 2D radiographs. Our approach requires a 3D generic jaw model and the cephalometric radiographs of two orthogonal views. Standard cephalometric radiographs were taken with a $165 \mathrm{~cm}$ tube-to-film distance, and $15 \mathrm{~cm}$ patient midsagittal plane-to-film distance, the magnification ration is $1.1 \%$.

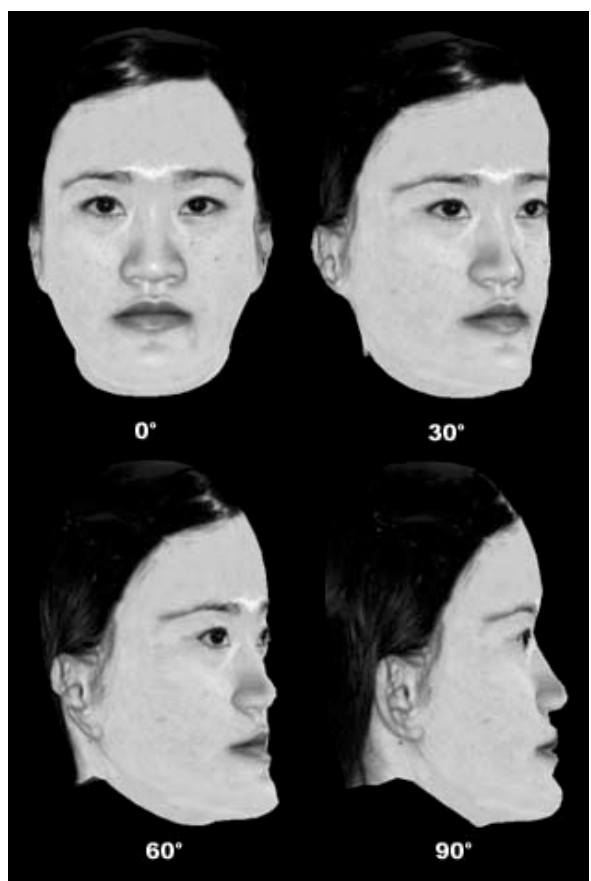

Figure 5. Different views of individualized texture mapped 3D head model

\subsection{Generic Jaw Model and Jaw Feature Vertices}

To generate an individualized jaw model, we first construct a generic jaw model, which contains 2776 polygons and 1450 vertices. The number of feature vertices used depends on the user acceptance level. The more the number of the feature vertices, the more accurate the model will be generated. However, the increase in the number of feature vertices will also increase the time required and the complexity for the user to define the surface contour of the jaw. As shown in Figure 6, 46 feature vertices are defined for the front view and 33 feature vertices are defined for the side view.

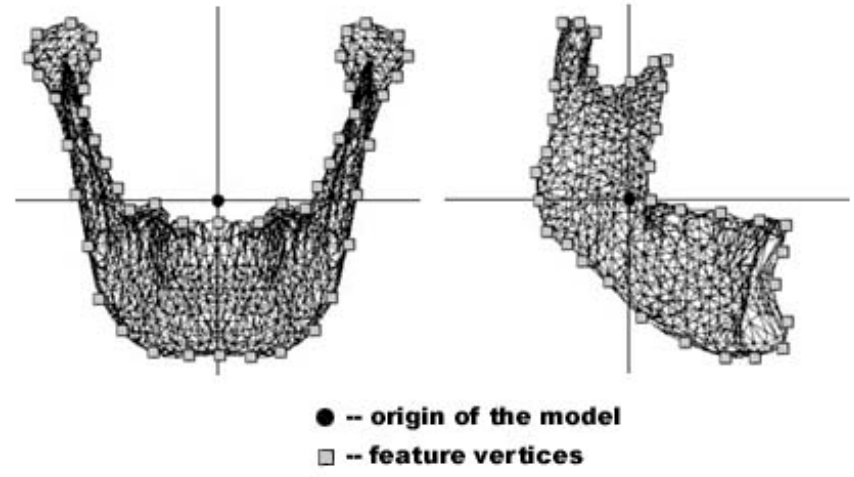

Figure 6. Front view and side view of generic jaw model 


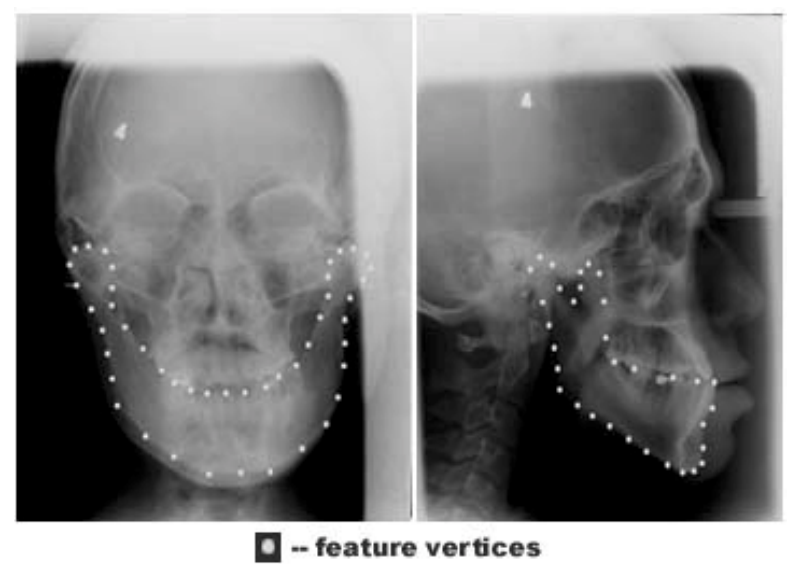

Figure 7. Feature vertices marked on the front view and side view of the radiographs

Figure 7 shows the feature vertices identified by the user manually according to the feature vertices definitions of the generic jaw model. It typically requires 1 minute to mark all the vertices for both views of the radiographs.

\subsection{Computation of Interval Points between Jaw Feature Vertices}

As mentioned above, the accuracy of the resulting jaw model is directly related to the number of feature vertices. However, the time required and the complexity for the user to define the feature vertices will also be increased. In order to increase the accuracy of the model generated without increasing the complexity of defining feature vertices, we have developed algorithm to find the interval points between every two feature vertices so as to increase the number of feature vertices. Thus, the number of feature vertices can be increased automatically. The equation used is shown as follows.

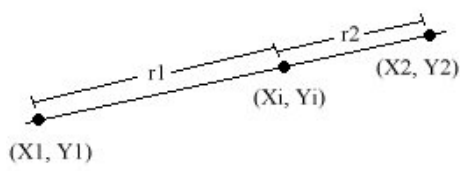

$X i=\frac{r_{1}^{*} X_{2}+r_{2} * X_{1}}{r_{1}+r_{2}} \quad Y i=\frac{r_{1}^{*} Y_{2}+r_{2} * Y_{1}}{r_{1}+r_{2}}$

where

$\mathrm{X}_{1}, \mathrm{Y}_{1}=$ coordinates of first feature vertex

$\mathrm{X}_{2}, \mathrm{Y}_{2}=$ coordinates of second feature vertex

$\mathrm{X}_{\mathrm{i}}, \mathrm{Y}_{\mathrm{i}}=$ coordinates of interval points

$r_{1}, r_{2}=$ ratio between interval points and feature vertices
After applying the above equation, the vertices on the surface contour of jaw model will be multiplied and all these vertices will be considered as the feature vertices of the jaw model afterwards. Figure 8 shows some of the results of the derived interval points on the front view of the radiograph.

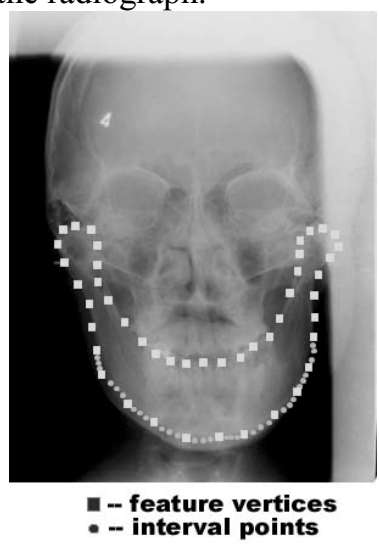

Figure 8. Front view of radiograph with feature vertices and derived interval points

Besides finding the interval points between feature vertices on the radiographs, the same technique can also be applied to find the same number of interval points between feature vertices on the generic jaw model.

\subsection{Individualized 3D Jaw Model Construction}

After defining the feature vertices for a jaw model, for each view of the jaw, a set of displacement vectors will be calculated by matching the feature vertices on the radiographs to those vertices of the generic jaw model as discussed in Section 2.4.

After calculating all the DVs, the new 2D coordinates for every vertex on the frontal and lateral views are generated. The resulting $2 \mathrm{D}$ polygonal models of the two views are superimposed on to the radiographs respectively as shown in Figure 9.

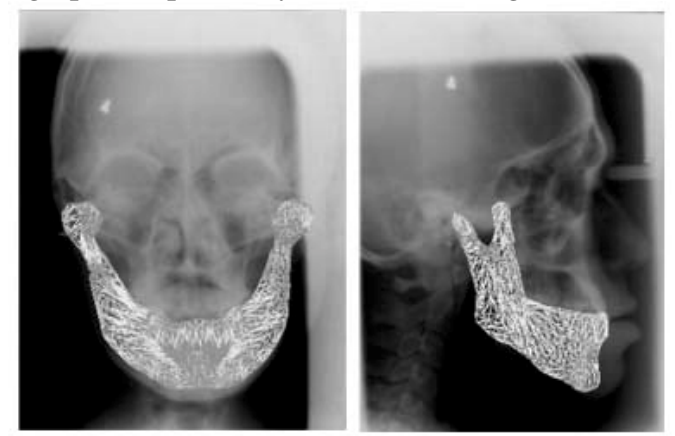

Figure 9. Alignment of the computer generated individualized jaw model and the radiographs of a patient 
Given the two sets of 2D coordinates of the computer constructed jaw model for the frontal and lateral views, the individualized 3D jaw model can then be generated as discussed in Section 2.5. Figure 10 shows the computer constructed jaw model for the patient in arbitrary views.
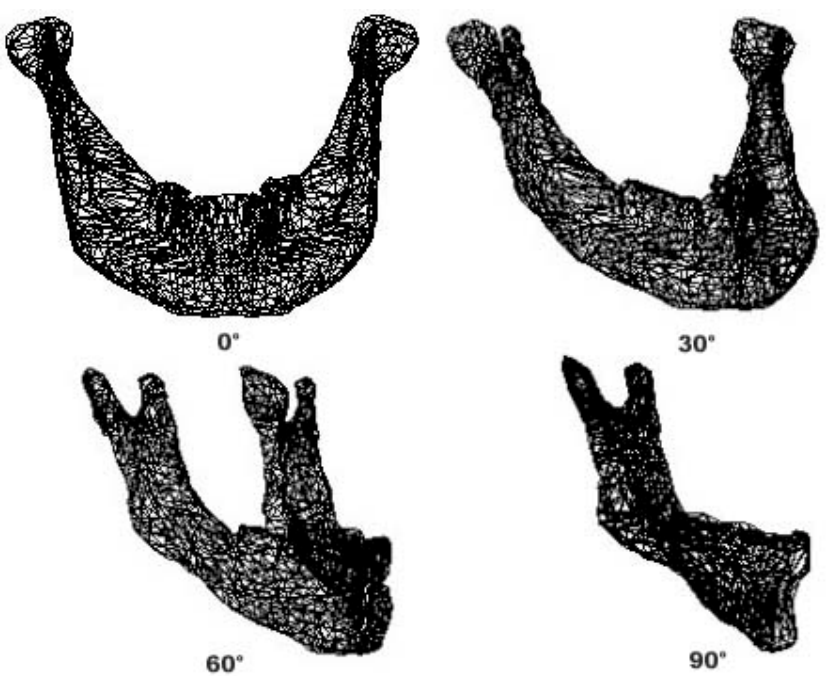

Figure 10. Different views of individualized 3D jaw model

\section{Combination of Head Model and Jaw Model for Surgical Planning}

After generating the head model and jaw model for the patient, the head model and jaw model can be combined to form a simulated patient as shown in Figure 11. With this simulated patient, the relationship and movement ratio between the soft and hard tissues can be defined. When an orthognathic surgery simulation is carried out, the transposition of a skeletal segment will cause the changes of the affected area of soft tissue according to the movement ratio. Thus, the post-operative effect of the patient can be predicted.

\section{Conclusion}

This paper proposed a semi-automatic method for generating a simulated patient with the use of portraits and radiographs of two orthogonal views for orthognathic surgery. This approach eliminates the need of costly imaging (data acquisition) modalities such as CT or MRI imaging and allows orthognathic surgery to be planned on low-cost platforms. This approach typically allows a textured 3D simulated patient to be constructed an synthesized in arbitrary views within 3 minutes on a PC-based platform. More
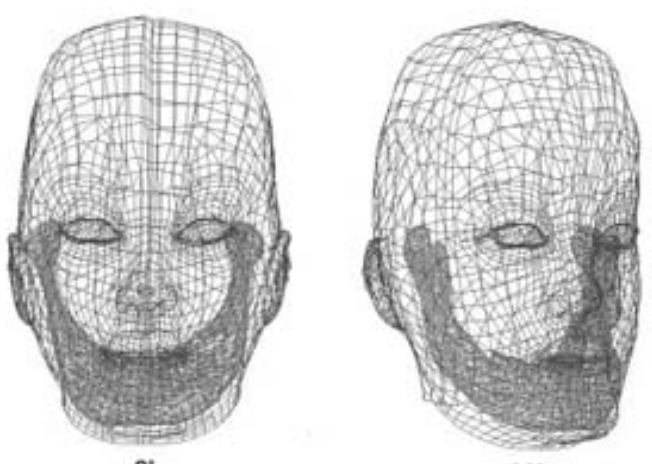

$0^{*}$

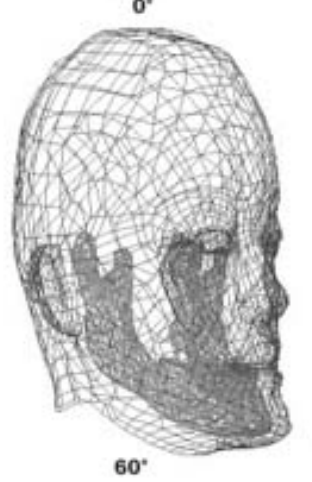

$30^{\circ}$

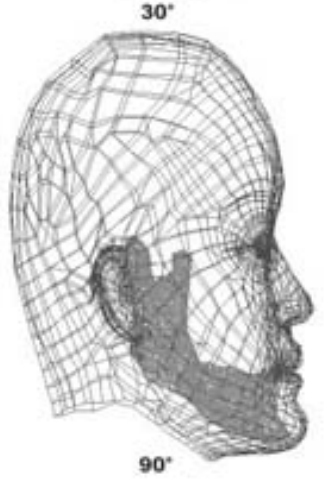

Figure 11. Different views of individualized 3D jaw model

importantly, to our knowledge, this is the first approach for constructing $3 \mathrm{D}$ hard tissue model such as jaw bone structures using conventional 2D radiographs as the data source.

Our technique can be generalized to handle a generic head model and jaw model of any number of polygons. A more accurate head model can be produced by using a generic model with more polygons. However, the increase in the number of polygons will also increase the computation time. Our algorithm supports portraits of multiple views for asymmetric face. For example, portraits of front view, left view and right view can be used in conjunction.

This simulated patient can be applied in a surgical planning system for Orthognathic Surgery, which is under development. With a special eyewear that is used to produce the stereoscopic images, the user can be immersed in the virtual reality environment for surgical planning with this simulated patient.

\section{References}

[1] Magnenat-Thalmann N, Thalmann D, "The direction of synthetics actors in the film Rendex-vous à Montr 1 , IEEE comp Graph Appl, 1987, 7:9-12. 
[2] Horace H.S. Ip, Lijun Yin, "Constructing a 3D individualized head model from two orthogonal views", The Visual Computer, 1996, 12:254

[3] James Jiong Xia, “Three-dimensional Surgical Planning and Simulation System for Orthognathic Surgery in Virtual Reality Environment", PhD Thesis.

[4] Paouri A, Magnenat-Thalmann N, Thalmann D, "Creating realistic three-dimensional human shape characters for computer-generated filems", Proceedings of Computer Animation'91, Springer, Tokyo, 1991, 89-99.

[5] Terzopolulous D, Waters K. "Analysis and synthesis of facial image sequences using physical and anatomical models", IEEE Trans Patt Anal Machine Intell, 1993, 15:569-579.
[6] Mason Woo, Jackie Neider, Tom Davis, "OpenGL Programming Guide Second Edition - The Official Guide to Learning OpenGL, Version 1.1", Addison-Wesley Developers Press, 1996.

[7] Clinical Department of Oral and Maxillofacial Surgery, University Hospital, Graz, Austria, "Dentofacial Planner Plus Version 1.5." Dentofacial Planner Plus Release Notes.

[8] CephScan Professional Software Products, Inc. "What is Cephalometric Analysis?" Available at $<$ http://www.cephscan.com/Product/product.htm>

[9] Swiss Federal Institute of Technology (ETH), Zürich, Department of Maxillofacial Surgery, "Simulating Facial Surgery Using Finite Element Models", SIGGRAPH 96 Conference Proceedings, Aug 1996, p421-428. 